Alma Mater Studiorum - Università di Bologna DEPARTMENT OF ECONOMICS

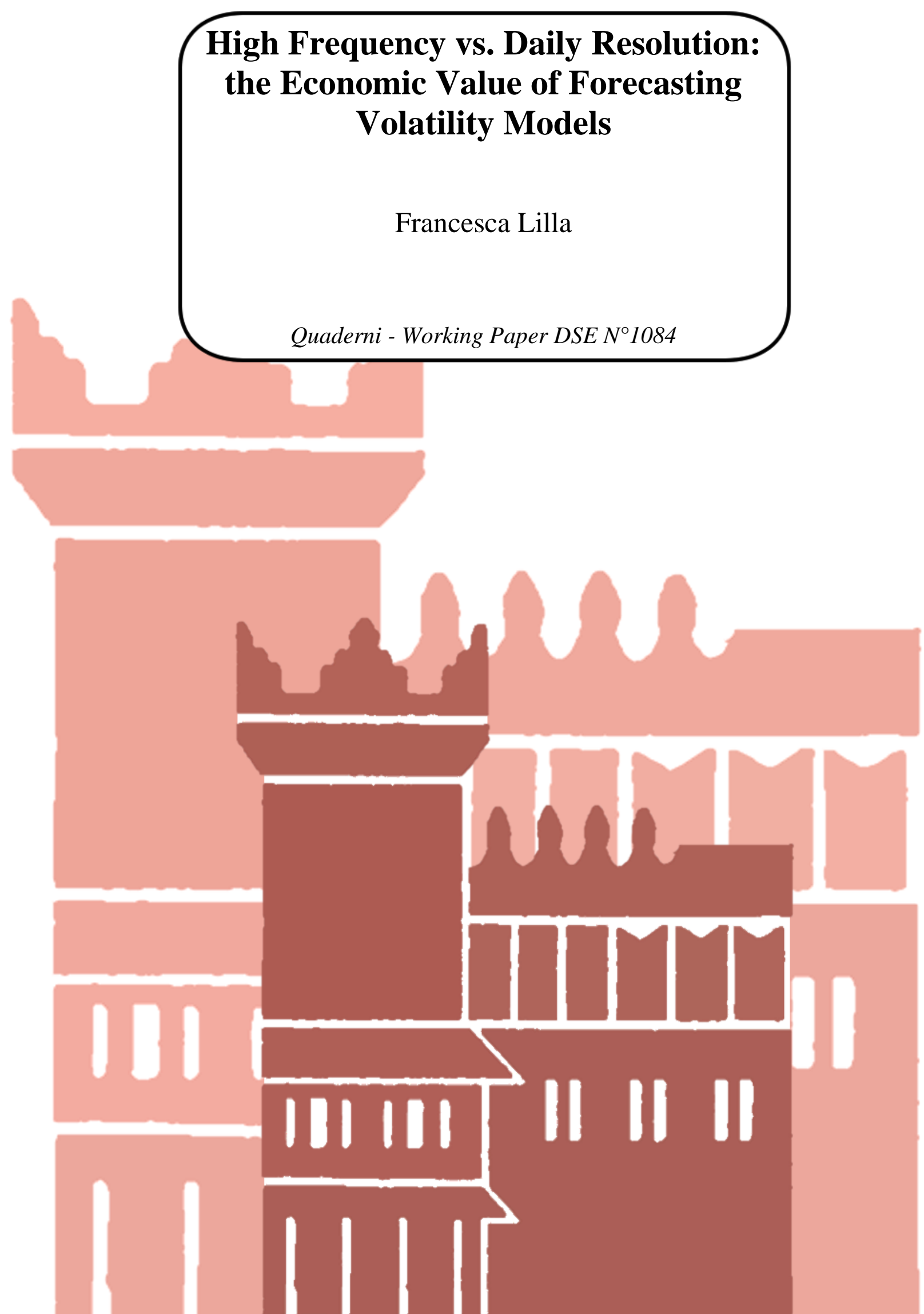




\title{
High Frequency vs. Daily Resolution: the Economic Value of Forecasting Volatility Models
}

\author{
Francesca Lilla *
}

November 15, 2016

\begin{abstract}
Forecasting-volatility models typically rely on either daily or high frequency (HF) data and the choice between these two categories is not obvious. In particular, the latter allows to treat volatility as observable but they suffer of many limitations. HF data feature microstructure problem, such as the discreteness of the data, the properties of the trading mechanism and the existence of bid-ask spread. Moreover, these data are not always available and, even if they are, the asset's liquidity may be not sufficient to allow for frequent transactions. This paper considers different variants of these two family forecasting-volatility models, comparing their performance (in terms of Value at Risk, $\mathrm{VaR})$ under the assumptions of jumping prices and leverage effects for volatility. Findings suggest that GARJI model provides more accurate VaR measures for the S\&P 500 index than RV models. Furthermore, the assumption of conditional normality is shown to be not sufficient to obtain accurate risk measures even if jump contribution is provided. More sophisticated models might address this issue, improving VaR results.
\end{abstract}

JEL-Classification: C58 C53 C22 C01 C13

Keywords: GARCH, DCS, jumps, leverage effect, high frequency data, realized variation, range estimator, $\mathrm{VaR}$

${ }^{*}$ University of Bologna, Department of Economics; Piazza Scaravilli 2, 40126 Bologna, Italy.

E-mail: francesca.lilla3@unibo.it 


\section{Introduction}

Modelling and forecasting volatility of asset returns are crucial for many applications, such as asset pricing model, risk management theory and portfolio allocation decisions. An earlier literature, including Engle (1982) and Bollerslev (1986) among others, has developed models of asset-volatility dynamics in discrete time, known as heteroscedastic volatility models, i.e. ARCH-GARCH. Thanks to the availability of high frequency (HF) data, a new strand of literature has originated a new class of models based on the Realized Volatility (RV) estimator, therefore introducing a non-parametric measure of return volatility (see Andersen et al., 001a, Barndorff-Nielsen, 2002 and Andersen et al., 2012). As a main innovation, RV models provides an ex-post observation of volatility, at odds with the standard ARCHGARCH approach, that treats volatility as a latent variable. Although forecasting-volatility models based on HF data are getting more and more popular in the literature, the choice between HF-data and daily-data models is yet not obvious, in particular from an empirical standpoint. In particular, the former still suffer of various limitations, that can be addressed only at the cost of an heavy manipulation of the original data. One of the main issue is the presence of the market microstructure noise, which prevents from getting a perfect estimate (at the limit) of the returns' variance (see Hansen and Lunde, 2006 and Aït-Sahalia et al., $2005,2011)$. The market microstructure noise may originate from different sources, including the discreteness of the data, the properties of the trading mechanisms and the existence of a bid-ask spread. Regardless of the source, when return from assets are measured based on their transaction prices over very tiny time-intervals, these measures are likely to be heavily affected by the noise and therefore brings little information on the volatility of the price process. Since the level of volatility is proportional to the time interval between two successive observations, as the time interval increases, the incidence of the noise remains constant, whereas the information about the "true" value of the volatility increases. Therefore, there is a trade-off between high frequency and accuracy, which has led authors to identify an optimal sampling frequency of 5 minutes $^{1}$. HF data also features another inconvenient: they are not always available and, even if they are, the asset may be not liquid enough to be frequently traded. On the contrary, daily data are relatively simple to record and collect, and are commonly easy-to-get. This paper shed light on the choice between HF-data and daily-data models, by assessing the economic value of the two family models, based on a comparison of

\footnotetext{
${ }^{1}$ Since the best remedy for market microstructure noise depends on the properties of the noise, if data sampled at higher frequency, e.g. tick-by-tick, are used the noise term needs to be modeled and, as far as I know, there is no unified framework about how to deal with it. Aït-Sahalia et al. (2005) define a new estimator, Two Scales Realized Volatility (TSRV), which takes advantages of the rich informations of tick-by-tick data and corrects the effects of microstructure noise on volatility estimation. The authors, instead of sampling over longer time horizon and discarding observations, make use of all data and model the noise as an "observation error". But the microstructure noise modeling goes beyond the scope of this work.
} 
their performance in forecasting asset volatility. Following the risk management perspective, I use value at risk (VaR) as the econometric metric of volatility forecastability, as suggested by Christoffersen and Diebold (2000). VaR is defined as the quantile of the conditional portfolio distribution, and is therefore quite intuitive as a measure: indeed, it is the most popular quantitative measure of the market risk associated to a portfolio of assets, and is generally adopted by banks and required by regulators all over the world ${ }^{2}$. In running the comparison between HF-data and daily-data models, this paper introduces two key assumptions. Firstly, the data generating process for asset prices features discontinuities in its trajectories, jumps ${ }^{3}$. Secondly, volatility (i.e. the conditional variance of asset returns) reacts differently to changes in asset return which have the same magnitude, but different sign, leverage effect. These two assumptions represent the main novelty of this paper, since none of the previous studies on the economic value of different forecasting-volatility models has investigated the matter under both jumping prices and leverage effect combined together. In the choice of the model to use for the comparison, I consider the GARJI model of Maheu and McCurdy (2004), as the baseline for the daily-data models. The latter is a mixed-GARCH jump model which allows for asymmetric responses to past innovations in asset returns: the news impact (resulting in jump innovations) may have a feedback effect on the expected volatility, in addition to the feedback effect associated with the normal error term. For the case of HF data, I consider models in which Realized Volatility (RV) is decomposed into continuous and discontinuous volatility components. The continuous component is captured by means of the bi-power variation (BV), introduced by Barndorff-Nielsen and Shephard (2004), whereas the discontinuous component (JV) is obtained as the difference between RV and BV at given point in time ${ }^{4}$. In Andersen et al. (2007), JV is obtained considering only jumps that are

\footnotetext{
${ }^{2}$ Banks often construct VaR from historical simulation (HS-VaR): VaR is the percentile of the portfolio distribution obtained using historical asset prices and today weigths. This procedure is characterized by a slow reaction to market conditions and for the inability to derive the term structure of VaR. The VaR term structure explains how risk measures vary across different investment horizons. In HS-VaR, for example, if T-day $1 \%$ VaR is calculated, the 1 -day $1 \% \mathrm{VaR}$ is simply scaled by $\sqrt{T}$. This relation is valid only if daily return are i.i.d. realizations of a Normal distribution. We know that is not the case since returns presents leptokurtosis and asymmetry. The main limit of HS-VaR is the substitution of the conditional return distribution with the unconditional counterpart. Risk Metrics and GARCH models represent improvements over HS-VaR measure. Both of them provide an explicit assumption about the DGP and the conditional variance but they have also important differences. In addition to the estimation method: GARCH conditional volatility is estimated by maximizing the log-likelihood function while the parameters used in Risk Metrics are chosen in an ad hoc fashion, they differ for the possibility to account for the term structure of VaR. This is because GARCH process allows for mean reversion in volatility while Risk Metrics does not, reproducing a flat term structure for VaR.

${ }^{3} \mathrm{~A}$ continuos price process is a restrictive assumption since it is not possible to distinguish between the dynamic originated from the the two sources of variability, i.e. continuos and discontinuous movements with consequences on the return generating process

${ }^{4}$ As shown in Andersen et al. (2002), Andersen et al. (2007), RV is a consistent estimator for the quadratic variation, whereas BV represents a consistent estimator of the continuous volatility component, i.e. the so-called integrated volatility, in the presence of jumping prices.
} 
found to be significative, and neglecting the others ${ }^{5}$. Corsi et al. (2010) consider instead all jumps, stressing the importance to correct the positive bias in BV due to jumps classified as consecutive. In this paper, I consider both these approaches and make a comparison among them, finding non conclusive evidence of better performance of one over the other. To account for the leverage effect, I introduce in this class of models the heterogeneous structure proposed by Corsi and Renó (2009).

Throughout this paper, the GARJI-VaR measures are obtained by following Chiu et al. (2005), that is, by adjusting for skewness and fat tails in the specification of the conditional distribution of returns ${ }^{6}$. The HF-VaR measures, instead, are computed by assuming a conditional Gaussian distribution for asset returns: as shown in Andersen et al. (2010), returns standardized for the square root of RV are indeed approximatively $\mathrm{Normal}^{7}$. In order to assess the models capability to forecast future volatility, I implement a back-testing procedure based on both the Christoffersen (1998) test and the Kupiec (1995) test. In addition to comparing the economic value of daily-data and HF-data models, the analysis performed in this paper sheds light on two other issues. The first is represented by the economic value per se, i.e. out of the comparison, of the class of forecasting volatility models adopting HFdata. This is done by considering different specifications of this family models. I first run a comparison among them (based on their forecasting performances); then, I compare some of them with their variant, obtained by using the Range estimator (RA) of Parkinson (1980). The choice of this particular benchmark is motivated by the fact that the RA estimator is likely to deliver a measure of volatility which lies in the middle between the measure obtained from HF estimators and that obtained from daily-data models ${ }^{8}$. My findings suggest that none of the HF-data models reviewed in this paper stands out from the others in term of forecasting capability. The second by-product of my analysis is a quantitative assessment of the importance of the explicit jump component in the conditional distribution of asset returns ${ }^{9}$.

\footnotetext{
${ }^{5}$ The authors with significant jumps refer to large value of $R V_{t}-B V_{t}$ while small positive values are treated both as part of continuous sample path variation or as measurement errors.

${ }^{6}$ The computation of VaR measure requires, in addition to the conditional volatility dynamics, the specification of the conditional distribution of returns.VaR is a conditional risk measure so an assumption on the conditional distribution of returns is needed. Conditional normality is an acceptable assumption (returns standardized by their conditional volatility could be approximately Gaussian even if the unconditional returns are not Gaussian) only if the volatility model is able to fatten conditionally Gaussian tails enough to match the unconditional distribution. If this is not the case another conditional distributional assumption is necessary.

${ }^{7}$ This result is confirmed by the standardized returns of the sample used in this paper. See Section 3.

${ }^{8}$ The RA estimator exploits information on the highest and the lowest price recorded in a given day for a particular asset. In this respect, it requires information on the intra-day activity (going beyond the simple closing price of the asset), but without relying on further information, that might be not ready available).

${ }^{9}$ The presence of a jump component is justified both at theoretical and empirical level. From a theoretical perspective, an explicit discontinuous volatility-component allows to have information on the market response to outside news, which is key for many applications. From an empirical standpoint, instead, it is very dif?cult to distinguish power-type tails from exponential-type tails, given that is not clear to what extent the return distribution is heavily tailed. In this regard, the jump component of a jump-diffusion model may be interpreted
} 
This point is addressed for both the family models considered in this paper. Hence, I first compare the forecasting volatility performances of each HF-data model with and without a decomposition of the RV into the continuous and the discontinuous component. Then, I run a similar analysis for the case of the daily-data models, considering the GARCH-t model as well as the Beta-t model ${ }^{10}$ proposed by Harvey and Luati (2014). According to my analysis, introducing an explicit, persistent jump component in the conditional return dynamics (together with an asymmetric response to bad and good news into conditional volatility dynamics) may help to forecast the ex-post volatility dynamics and obtain more accurate VaR measures, but only for the case of daily-data models. For HF-data models, accounting for jumping prices does not seem to improve significantly the accuracy of the estimates. The rest of the paper is organized as follows. Section 2 describes the most related literature and the main contributions of this paper. Section 3 summarizes the volatility measures and the forecasting models based on both HF and daily data. In this Section are also presented the estimated parameters based on the entire sample. Section 4 and Section 5 show, respectively, the backtesting methods used to evaluate forecasting models accurancy and the emprirical results. Section 6 concludes.

\section{Literature}

In this Section, I will briefly present the most related papers to mine. As far as I know, the closest papers to this work are Giot and Laurent (2004), Clements et al. (2008) and Brownlees and Gallo (2010). All of these works investigate high frequency measures in a VaR framework but the scope is different from what I want to asses in the present paper. Precisily I want to understand, from an economic point of view, if forecasting volatility with models based on high frequency data or with models based on daily data deals with the same VaR accurancy. Moreover I focus on the role of jump component in both types of models and I am able to compare VaR accuracy in each subgroup of data specification. I try to asses this point comparing forecasting models based on RV measures, its decomposition in BV e JV and a cascade dynamics for leverage effect with daily based models assuming an explicit jump component or fat tails in the conditional returns dynamics. In particular, Giot and Laurent (2004) compare the performance of a daily ARCH-type model with the performance of a model based on the daily RV in a VaR framework. Even if the scope of this paper is strictly related to the goal of the present work, the models chosen are not the same. The present paper tries to

as the market response to outside news: when good or bad news arrive at a given point in time, the asset price changes according to the jump size (and the jump sign) and an extreme sources of variation is added to the idyosincratic component.

${ }^{10}$ Beta- $t$ model belongs to the genral class of Dynamic Conditional Score (DCS) model. They are also known as Generalized Autoregressive Score (GAS) model proposed by Creal et al. (2013). 
consider all the most recent research about volatility forecasting, belonging to two groups of sampling data, assuming two fundamental stylized facts about returns: jumps and leverage effect. Giot and Laurent (2004) find that VaR specification based on RV does not really improve on the performance of a VaR model estimated using daily returns. The key issue is to use a model that clearly recognizes and takes into account the key features of the empirical data. Clements et al. (2008) evaluate quantile forecasts focusing exclusively on models based on RV and they try to explain factors that appear to give good HF quantile forecast of exchange rates ${ }^{11}$. The authors do not provide leverage effect in volatility dynamics. Even if the scope is different and the specifications are not the same, Clements et al. (2008) underline an important result: the hypothesis on expected future returns. The distributional assumption for expected future returns is needed for computing quantile irrespective of the frequency of data used. Brownlees and Gallo (2010) forecast VaR using different volatility measures based on ultra-high-frequency data using a two-step VaR prediction procedure. This paper differs from mine in terms of volatility dynamics specifications, VaR forecasting procedure and accuracy. Moreover, the authors model close-to-close returns with a Student's $t$ distribution and estimate some alternative models based on daily returns only for completeness. In light of this literature, this paper comes from the observation that great deal of the work has exploited only specific volatility model without allowing for a complete comparison between daily and HF based models. Therefore, the analysis conducted in this work aims to contribute to this literature filling this gap. In sum, this paper contributes to the existing literature assessing the economic usefulness of HF and daily data and trying to understand the importance of the distributional assumption for expected future returns in terms of VaR accurancy. This paper would be a point of debate and a possible link between findings by academic and economic requirements of practitioner communities.

\section{Volatility Measures and Forecasts}

\subsection{Estimates of volatility with High Frequency Data}

The RV measure is an estimator for the total quadratic variation, namely it converges in probability, as the sampling frequency increases, to the continuos volatility component if there are no jumps while it converges to the sum of continuos and discontinuous volatility components if at least one jump occurs. As explained in Andersen et al. (2012), it is possible to use the daily RV measures, the ex-post volatility observations, to construct the ex-ante volatility forecasts. This is possible simply by using standard ARMA time series tools but it is important to take into account the difference with GARCH-type forecasting. The fun-

\footnotetext{
${ }^{11}$ Clements et al. (2008) wants also to understand if the results presented for stock returns can be carried over exchange rates.
} 
damental difference is that in the former case the risk manager treats volatility as observed while in the latter framework volatility is inferred from past returns conditional on a specific model. The idea behind the RV is the following: as we know prices are not available on continuous basis but with prices recorded at higher frequency than daily, say, every minute, a daily RV could easily be computed from one-minute squared returns. In this way the "true" ex-post volatility for the day $t$ can be considered as observable.

More precisely, the RV on day $t$ based on returns at the $\Delta$ intraday frequency is

$$
R V_{t}(\Delta) \equiv \sum_{j=1}^{N(\Delta)} r_{t, j}^{2}
$$

where $r_{t, j}=p_{t-1+j \Delta}-p_{t-1+(j-1) \Delta}$ and $p_{t-1+j \Delta}$ is the log-price at the end of the $j t h$ interval on day $t$ and $N(\Delta)$ is the number of the observations available at day $t$ recorded at $\Delta$ frequency. In the absence of microstructure noise, as $\Delta \rightarrow 0$ the RV estimator approaches the integrated variance of the underlying continuous-time stochastic volatility process on day $t$ :

$$
R V_{t} \longrightarrow_{p} I V_{t} \quad \text { where } \quad I V_{t}=\int_{t-1}^{t} \sigma^{2}(\tau) d \tau
$$

Furthermore since in this paper I consider that the the underlying price process is characterized by discontinuities, the previous convergence is not valid but the RV estimators approaches in probability to the sum of the integrated volatility and the variation due to jumps that occurred on day $t$. It is equal to:

$$
R V_{t} \longrightarrow p \int_{t-1}^{t} \sigma^{2}(\tau) d \tau+\sum_{j=1}^{\zeta_{t}} J_{t, j}^{2}
$$

If jumps $\left(J_{t, j}\right)$ are absent, the second term vanishes and the realized volatility consistently estimates the integrated volatility. A nonparametric estimate of the continuous volatility component in the case of discontinuities in the price process is obtained by using the bipower variation $(\mathrm{BV})$ measures:

$$
B V_{t} \equiv \frac{\pi}{2} \frac{N(\Delta)}{N(\Delta)-1} \sum_{j=1}^{N(\Delta)-1}\left|r_{t, j}\right|\left|r_{t, j+1}\right|
$$

The idea behind this estimator is that when the $\Delta$ goes to zero the probability of jumps arriving both in time interval $j \Delta$ and $(j+1) \Delta$ goes to zero as $\left|r_{t, j}\right|$ so the product vanishes asymptotically. The notion of BV measures the behavior of adjacent returns. If returns are driven by some continuous martingale component, given that this component will appear in both increments, the product in BV will capture it. If there is an occasional jump the 
product will vanish, BV will not capture jump effect, since the probability to have jumps in two adjacent returns tends to zero. Hence, combining these results, the contribution to the total return variation stemming from the jump component $\left(J V_{t}\right)$ is consistently estimated by

$$
R V_{t}-B V_{t} \longrightarrow p \sum_{j=1}^{\zeta_{t}} J_{t, j}^{2}
$$

This intraday variation measure is used to separate the continuous and the jump component; the latter can be consistently estimated by the difference between RV and BV. Considering the suggestion of Barndorff-Nielsen and Shephard (2004) the empirical measurements are truncated at zero in order to ensure that all of the daily estimates are nonnegative:

$$
J V_{t}=\max \left\{R V_{t}-B V_{t}, 0\right\}
$$

According to Andersen et al. (2007), this truncation reduces the problem of measurement error with fixed sampling frequency but it captures a large number of nonzero small positive values in the jump component series. These small positive values can be treated both as part of the continuous sample path variation process or as measurement errors and, according to the authors, only large values of $R V_{t}-B V_{t}$ are associated with the jump component, i.e. "significant jumps".

In order to identify statistically significant jumps the authors suggest the use of the following statistic:

$$
Z_{t}=\frac{\log \left(R V_{t}\right)-\log \left(B V_{t}\right)}{\sqrt{N(\Delta)^{-1}\left(\mu_{1}^{-4}+2 \mu_{1}^{-2}-5\right) T Q_{t} B V_{t}^{-2}}} \longrightarrow_{d} N(0,1)
$$

where $\mu_{1}=\sqrt{2 / \pi}$. In the denominator appears the realized tripower variation (TQ) that is the estimator of the integrated quarticity as required for a standard deviation notion of scale:

$$
T Q_{t}=N(\Delta) \mu_{4 / 3}^{-3} \sum_{j=3}^{N(\Delta)}\left|r_{t, j}\right|^{4 / 3}\left|r_{t, j+1}\right|^{4 / 3}\left|r_{t, j+2}\right|^{4 / 3}
$$

where $\mu_{4 / 3}=2^{2 / 3} \Gamma(7 / 6) \Gamma(1 / 2)$. The significant jumps and the continuos component are identified and estimated respectively as:

$$
\begin{aligned}
& J V_{t}=\mathbb{1}_{\left\{Z_{t}>\Phi_{\alpha}\right\}}\left(R V_{t}-B V_{t}\right) \\
& C V_{t}=R V_{t}-J V_{t}=\mathbb{1}_{\left\{Z_{t} \leq \Phi_{\alpha}\right\}} R V_{t}-\mathbb{1}_{\left\{Z_{t}>\Phi_{\alpha}\right\}} B V_{t}
\end{aligned}
$$

where $\mathbb{1}$ is the indicator function and $\Phi_{\alpha}$ is the $\alpha$ quantile of a Standard Normal cdf. Corsi et al. (2010) show that the nonparametric estimator BV can be strongly biased in finite sample because of the presence of consecutive jumps and they define a new nonparametric estima- 
tor, called Threshold Bipower Variation (TBV). In particular, according to the authors TBV is able to correct for the positive bias of BV in the case of consecutive jumps:

$$
T B V_{t}=\mu_{1}^{-2} \sum_{j=2}^{N(\Delta)}\left|r_{t, j}\right|\left|r_{t, j+1)}\right| \mathbb{1}_{\left\{\left|r_{t, j}\right|^{2}<\theta_{j}\right\}} \mathbb{1}_{\left\{\left|r_{t, j+1}\right|^{2}<\theta_{j+1}\right\}}
$$

where $\theta$ is strictly positive random threshold function equal to $\hat{V}_{t} c_{\theta}^{2}, \hat{V}_{t}$ is an auxiliary estimator and $c_{\theta}^{2}$ is a scale-free constant that allows to change the threshold. The jump detection test presented by Corsi et al. (2010) is the following:

$$
\mathrm{C}-\mathrm{Tz}=N(\Delta)^{-1 / 2} \frac{\left(R V_{t}-T B V_{t}\right) R V_{t}^{-1}}{\sqrt{\left(\frac{\pi^{2}}{4}+\pi-5\right) \max \left\{1, \frac{T T r i P V_{t}}{T B V_{t}^{2}}\right\}}} \longrightarrow_{d} N(0,1)
$$

where TTriPV is a quarticity estimator which is obtained by multiplying the TBV by $\mu_{4 / 3}^{-3}$. Also in this case the jumps and the continuos component are identified and estimated respectively as:

$$
\begin{aligned}
& J V_{t}=\mathbb{1}_{\left\{\mathrm{C}-\mathrm{Tz} z_{t}>\Phi_{\alpha}\right\}}\left(R V_{t}-\mathrm{TB} V_{t}\right) \\
& C V_{t}=R V_{t}-J V_{t}=\mathbb{1}_{\left\{\mathrm{C}-\mathrm{Tz} z_{t} \leq \Phi_{\alpha}\right\}} R V_{t}-\mathbb{1}_{\left\{\mathrm{C}-\mathrm{Tz} z_{t}>\Phi_{\alpha}\right\}} T B V_{t}
\end{aligned}
$$

The other measure chosen in this work is the Range volatility (RA) presented by Parkinson (1980):

$$
R A_{t}=\frac{1}{4 \log 2}\left(\log \left(H_{t}\right)-\log \left(L_{t}\right)\right)^{2}
$$

This estimator is constructed by taking the highest price $(\mathrm{H})$ and the lowest price $(\mathrm{L})$ for each day as summary of the intraday activity, i.e. the full path process. Its major empirical advantage is that for many assets these informations are ready available. The RA estimator, as RV, includes both continuos and discontinuous components and, as shown in Alizadeh et al. (2002), it is affected by a much lower measurement error and it is more robust to microstructure noise in a stochastic volatility framework and it allows to extract efficiently latent volatility.

\subsection{Forecasting volatility using High Frequency Data}

In the literature there is no consensus if jumps help to forecast volatility. In this sense this work can be useful in order to understand, in a VaR framework, if allowing for an explicit jump component is important to forecast volatility, independently of the sampling frequency of the price process. Moreover, if different sampling frequencies (daily and 5-minutes) are considered then a discrimination between the two kinds if data used, according to VaR forecasts, can be done.

For all forecasting models that I am going to describe in this section, I define a log spec- 
ification both for inducing normality and for ensuring positivity of volatility forecasts ${ }^{12}$. The natural starting point in forecasting volatility is to use an Autoregressive (AR) specification $^{13}$. The first model for both RV and RA is the AR model. In particular, an AR(8) model is identified for both RV measure and for Range estimator ${ }^{14}$. These specifications are easy to implement but they are not able to capture the volatility long-range dependence due to the slowly decaying autocorrelation of returns. As alternative it is possible to use the Heterogenous Autoregressive model proposed by Corsi (2009). This model can be seen as an approximation of long memory model, namely it exploits longer dependence but it is easier to implement than the pure long-memory model (see Andersen et al., 2007, Corsi and Renó, 2009). The second forecasting model for both volatility measures is the Heterogeneous Autoregressive model (HAR) that is able to capture serial dependence in volatility. The aggregate measures for the daily, weekly and monthly realized volatility are computed as sum of past realized volatilities over different horizons:

$$
R V_{t}^{(N)}=\frac{1}{N} R V_{t}+\cdots+R V_{t-N+1}
$$

where $\mathrm{N}$ is typically equal to 1,5 or 22 according to if the time scale is daily, weekly or monthly.

Then, HAR-RV becomes:

$$
\log R V_{t+h}=\beta_{0}+\beta_{1} \log R V_{t+h-1}+\beta_{2} \log R V_{t+h-1}^{(5)}+\beta_{3} \log R V_{t+h-1}^{(22)}+\epsilon_{t}
$$

where $\epsilon_{t}$ is IID zero mean and finite variance noise ${ }^{15}$

Moreover, as suggested in Corsi and Renó (2009), the heterogeneous structure applies also to leverage effect and, as a consequence, volatility forecasts are obtained by considering asymmetric responses of realized volatility not only to previous daily negative returns but also to their weekly and monthly components. So, the past aggregated negative returns are constructed as:

$$
l_{t}^{(N)}=\frac{1}{N}\left(r_{t}+\cdots+r_{t-N+1}\right) \mathbb{1}_{\left\{\left(r_{t}+\cdots+r_{t-N+1}\right)<0\right\}}
$$

\footnotetext{
${ }^{12}$ Volatility forecasts at each time is obtained by applying the exponential transformation.

${ }^{13}$ It is also possible to use an ARMA model to forecast volatility in order to consider some measurement errors since the empirical sampling is not done in continuous time.

${ }^{14}$ The identification procedure for the order of both AR models is done by exploiting the sample autocorrelation and the sample partial autocorrelation function, by running both AIC and BIC information criteria and significance of single parameters. Then I check the properties of the residuals: they are normal and the Ljung Box test does not reject the null of no autocorrelation at any significance level.

${ }^{15}$ Corsi and Renó (2009) model the dynamic of the latent quadratic variation, call it $\tilde{\sigma}_{t}$. Suppose that $\hat{V}_{t}$ is a generic unbiased estimator of $\tilde{\sigma}_{t}$ and $\log \left(\tilde{\sigma}_{t}\right)=\log \left(\hat{V}_{t}\right)+\omega_{t}$ where $\omega_{t}$ is a zero mean and finite variance measurement error. Then $\epsilon_{t}$ is independent from $\omega_{t}$.
} 
Then the L-HAR model is defined as:

$$
\begin{gathered}
\log R V_{t+h}=\beta_{0}+\beta_{1} \log R V_{t+h-1}+\beta_{2} \log R V_{t+h-1}^{(5)}+\beta_{3} \log R V_{t+h-1}^{(22)}+ \\
\beta_{4} l_{t+h-1}+\beta_{5} l_{t+h-1}^{(5)}+\beta_{6} l_{t+h-1}^{(22)}+\epsilon_{t}
\end{gathered}
$$

The explanatory variables of the HAR-RV model can be decomposed into continuous and jump components, in this way the forecasting model obtained is:

$$
\begin{aligned}
\log R V_{t+h}= & \beta_{0}+\beta_{1} \log C V_{t+h-1}+\beta_{2} \log C V_{t+h-1}^{(5)}+\beta_{3} \log C V_{t+h-1}^{(22)}+ \\
& \beta_{4} \log \left(1+J V_{t+h-1}\right)+\beta_{5} \log \left(1+J V_{t+h-1}^{(5)}\right)+\beta_{6} \log \left(1+J V_{t+h-1}^{(22)}\right)+\epsilon_{t}
\end{aligned}
$$

Depending on how the jump component is detected three different forecasted realized volatility are obtained. First, the HAR-Jumps is obtained according to (2) and for the continuos component to (1). Second, the HAR-CV-JV model is obtained following Andersen et al. (2007), namely according to (4). The last model, HAR-C-J is defined according to (6) following the estimation strategy presented in Corsi and Renó (2009). Also in the case of jumps the leverage variable is considered. In this way the three forecasting specifications used are obtained by adding to (12) the cascade leverage variables computed as in (10):

$$
\begin{aligned}
\log R V_{t+h}= & \beta_{0}+\beta_{1} \log C V_{t+h-1}+\beta_{2} \log C V_{t+h-1}^{(5)}+\beta_{3} \log C V_{t+h-1}^{(22)}+ \\
& \beta_{4} \log \left(1+J V_{t+h-1}\right)+\beta_{5} \log \left(1+J V_{t+h-1}^{(5)}\right)+\beta_{6} \log \left(1+J V_{t+h-1}^{(22)}\right)+ \\
& \beta_{7} l_{t+h-1}+\beta_{8} l_{t+h-1}^{(5)}+\beta_{9} l_{t+h-1}^{(22)}+\epsilon_{t}
\end{aligned}
$$

Referring to the previous consideration about the way of computing the two volatility component I obtain three new model specifications LHAR-Jumps, LHAR-CV-JV and LHAR-C-J. As explained in Section 2, RA, assuming that starting from daily data it sum up the trading information at a given day, performs remarkably well given its simplicity. Starting from the previous considerations and from the fact that RV and RA are both ex-post volatility measures I want to asses the forecast ability of the RA also according to the heterogeneity in the time horizons of investors in the financial market and considering the leverage effect. In this way I define two different forecasting models, in addition to the AR(8) model:

$$
\log R A_{t+h}=\beta_{0}+\beta_{1} \log R A_{t+h-1}+\beta_{2} \log R A_{t+h-1}^{(5)}+\beta_{3} \log R A_{t+h-1}^{(22)}+\epsilon_{t}
$$

called Range-HAR and

$$
\begin{gathered}
\log R A_{t+h}=\beta_{0}+\beta_{1} \log R A_{t+h-1}+\beta_{2} \log R A_{t+h-1}^{(5)}+\beta_{3} \log R A_{t+h-1}^{(22)}+ \\
\beta_{4} l_{t+h-1}+\beta_{5} l_{t+h-1}^{(5)}+\beta_{6} l_{t+h-1}^{(22)}+\epsilon_{t}
\end{gathered}
$$


called Range-L-HAR, where RA in computed as in (7).

\subsection{Forecasting volatility using daily data}

The first specification for the continuous volatility component is the GARJI model ${ }^{16}$ :

$$
\begin{aligned}
R_{t} & =\mu+\sigma_{t} z_{t}+\sum_{i=1}^{N_{t}} X_{t}^{(i)} \\
\lambda_{t} & =\lambda_{0}+\rho \lambda_{t-1}+\gamma \xi_{t-1} \\
\sigma_{t}^{2} & =\gamma+g\left(\Lambda, \mathcal{F}_{t-1}\right) \epsilon_{t-1}^{2}+\beta \sigma_{t-1}^{2} \\
g\left(\Lambda, \mathcal{F}_{t-1}\right) & =\exp \left(\alpha+\alpha_{j} E\left(N_{t} \mid \mathcal{F}_{t-1}\right)\right. \\
& \left.+\mathbb{1}_{\left\{\epsilon_{t-1}<0\right\}}\left[\alpha_{a}+\alpha_{a, j} E\left(N_{t} \mid \mathcal{F}_{t-1}\right)\right]\right)
\end{aligned}
$$

where $\epsilon_{t}=\epsilon_{1, t}+\epsilon_{2, t}=\sigma_{t} z_{t}+\sum_{i=1}^{N_{t}} X_{t}^{(i)}, z_{t} \sim \mathcal{N}(0,1), N_{t} \sim \operatorname{Poisson}\left(\lambda_{t}\right), X_{t}^{(j)} \sim \mathcal{N}\left(\mu, \omega^{2}\right)$ and $\xi_{t-1}=E\left[N_{t-1} \mid \mathcal{F}_{t-1}\right)-\lambda_{t-1}$.

As explained in Maheu and McCurdy (2004), the last equation allows for the introduction of a differential impact if past news are deemed good or bad. If past news are business as usual, in the sense that no jumps occurred, and are positive, then the impact on current volatility will be $\exp (\alpha) \epsilon_{t-1}^{2}$. If no jump took place but news are bad, the volatility impact becomes $\exp \left(\alpha+\alpha_{a}\right) \epsilon_{t-1}^{2}$. If a jump took place, with good news, the impact is $\exp \left(\alpha+\alpha_{j}\right) \epsilon_{t-1}^{2}$. If a jump took place, with bad news, then the impact becomes $\exp \left(\alpha+\alpha_{j}+\alpha_{a}+\alpha_{a, j}\right) \epsilon_{t-1}^{2}$. The arrival rate of jumps is assumed to follow a non homogeneous Poisson process while jump size is described by a Normal distribution. In this way the single impact of extraordinary news on volatility is identified through the combination of parameters in $g\left(\Lambda, \mathcal{F}_{t-1}\right)$. The idea of the authors is the following: the conditional variance of returns is a combination of a smoothly evolving continuous-state GARCH component and a discrete-jump component. In addition previous realization of both innovations, $\epsilon_{1, t}$ and $\epsilon_{2, t}$ affect expected volatility through the GARCH component of the conditional variance. This feedback is important because once return innovations are realized, there may be strategic or liquidity tradings related to the propagation of the news which are further sources of volatility clustering ${ }^{17}$. With this model it is possible to allow for several asymmetric responses to past returns innovations and then obtain a richer characterization of volatility dynamics, especially with respect to events in the tail of the distribution (jumps).

In particular $E\left[N_{t-1} \mid \mathcal{F}_{t-1}\right)$ is the ex-post assessment of the expected number of jumps that

\footnotetext{
${ }^{16}$ The ARJI specification is obtained by imposing $\alpha_{j}=\alpha_{a}=\alpha_{a, j}=0$

${ }^{17} \mathrm{~A}$ source of jumps to return can be important and unusual news, such as earnings surprise (result as an extreme movement in price) while less extreme movements in price can be due to typical news events, such as liquidity trading and strategic trading.
} 
occurred from $t-2$ to $t-1$ and it is equal to $\sum_{j=0}^{\infty} j P\left(N_{t-1}=j \mid \mathcal{F}_{t-1}\right)$. Therefore $\xi_{t-1}$ is the change in the econometrician's conditional forecast on $N_{t-1}$ as the information set is updated, it is the difference between the expected value and the actual one. As shown by Maheu and McCurdy (2004) this expression may be inferred using Bayes' formula:

$$
P\left(N_{t}=j \mid \mathcal{F}_{t-1}\right)=\frac{f\left(R_{t} \mid N_{t}=j, \mathcal{F}_{t-1}\right) P\left(N_{t}=j \mid \mathcal{F}_{t-1}\right)}{f\left(R_{t} \mid \mathcal{F}_{t-1}\right)} \quad \text { for } \quad j=0,1,2, \ldots
$$

Indeed, conditional on knowing $\lambda_{t}, \sigma_{t}$, and the number of jumps that took place over a time interval, $N_{t}=j$, the density of $R_{t}$ in terms of observable is Normal:

$$
f\left(R_{t} \mid \mathcal{F}_{t-1}\right)=\sum_{j=0}^{\infty} f\left(R_{t} \mid N_{t}=j, \mathcal{F}_{t-1}\right) \times P\left(N_{t}=j \mid \mathcal{F}_{t-1}\right)
$$

where

$$
f\left(R_{t} \mid N_{t}=j, \mathcal{F}_{t-1}\right)=\frac{1}{\sqrt{2 \pi\left(\sigma_{t}^{2}+j \delta^{2}\right)}} \exp \left(-\frac{\left(R_{t}-\mu+\theta \lambda_{t}-\theta j\right)^{2}}{2\left(\sigma_{t}^{2}+j \delta^{2}\right)}\right)
$$

Naturally the likelihood function is defined starting from (22), where $\tilde{\theta}$ is the vector of the parameters of interest, i.e. $\tilde{\theta}=\left(\gamma, \rho, \theta, \delta^{2}, \alpha, \alpha_{j}, \alpha_{a}, \alpha_{a j}, \omega, \beta, \lambda_{0}, \mu\right)$ :

$$
\mathcal{L}\left(R_{t} \mid N_{t}=j, \mathcal{F}_{t-1} ; \tilde{\theta}\right)=\prod_{t=1}^{T} f\left(R_{t} \mid N_{t}=j, \mathcal{F}_{t-1}\right)
$$

and the log-likelihood is:

$$
l\left(R_{t} \mid N_{t}=j, \mathcal{F}_{t-1} ; \tilde{\theta}\right)=\sum_{t=1}^{T} \log f\left(R_{t} \mid N_{t}=j, \mathcal{F}_{t-1}\right)
$$

In order to deal with the infinite summation in the likelihood and in the filter (20), I adopt $N_{t}=10$ because the conditional Poisson distribution has almost zero probability in the tails for values of $N_{t} \geq 10$, as suggested in Maheu and McCurdy (2004). Even if this is not the focus of this paper, GARCH- $t$ model and Beta-t-GARCH model for conditional volatility are chosen in order to understand if GARJI model can provide a better fit to the empirical distribution of the data and a better quantile forecast with respect to volatility specifications based on fat tails, such as $t$-Student. Beta-t-GARCH model consists of an observation driven model based on the idea that the specification of the conditional volatility as a linear combination of squared observations is taken for granted but the consequences are that it responds too much to extreme observations and the effect is slow to dissipate. So, the authors define a model in which the observation are generated by a conditional heavy tailed distribution with time varying scale parameters and where the dynamics rare driven by the score of the conditional distribution. In this way the model counts the innovation outliers but also the 
additive outliers.

\section{Computing and comparing VaR forecasts}

The predicted VaRs are based on the predicted volatility and they depend on the assumption on the conditional density of daily returns. The one day-ahead VaR prediction at time $t+1$ conditional on the information set at time $t$ is:

$$
\widehat{V a R}_{t+1 \mid t}=\sqrt{\widehat{\sigma}_{t+1 \mid t}^{2}} F_{t}^{-1}(\alpha)
$$

In (25) $\widehat{\sigma}_{t+1 \mid t}^{2}$ is the returns variance, estimated in both parametric and non-parametric models, $F_{t}^{-1}(\alpha)$ is the inverse of the cumulative distribution of daily returns while $\alpha$ indicates the degree of significance level. In the case of HF data $\widehat{\sigma}_{t+1 \mid t}^{2}$ is equal to $\widehat{R V}_{t}$ or $\widehat{R A}_{t}$ estimated as explained in the section 3.2 while for GARJI model the returns variance is not simply the modified GARCH dynamic but it also consist of the variance due to jumps (Hung et al., 2008):

$$
\widehat{V a R}_{t+1 \mid t}=\sqrt{\widehat{\sigma}_{t+1 \mid t}^{2}+\left(\widehat{\theta}_{t}^{2}+\widehat{\delta}_{t}^{2}\right) \widehat{\lambda}_{t}} \widetilde{F}_{t}^{-1}(\alpha)
$$

where $\widetilde{F}_{t}^{-1}(\alpha)=F_{t}^{-1}(\alpha)+\frac{1}{6}\left(\left(F_{t}^{-1}(\alpha)\right)^{2}-1\right) S k\left(R_{t} \mid t \mathcal{F}_{t-1}\right)$ and $S k\left(R_{t} \mid t \mathcal{F}_{t-1}\right)$ is the conditional return skewness computed after estimating the model. Once obtained VaR forecasts, I asses the relative performance of the models through the violation ${ }^{18}$ rate and the quality of the estimates by applying backtesting methods ${ }^{19}$.

A violation occurs when a realized return is greater than the estimated return. The violation rate is defined as the total number of violations divided by the total number of one periodforecasts ${ }^{20}$. The tests used in this paper are the Unconditional Coverage and Conditional

\footnotetext{
${ }^{18}$ In the testing literature exception is used instead of violation because the former is referred, as I explain later, to a loss function. The loss function changes according to the test applied and the motivation behind the testing strategies.

${ }^{19}$ The backtesting tests give the possibility to interpret the results and then the quality of the forecasting model choose in inferential terms.

${ }^{20}$ As well explained in Gençay et al. (2003) at $q$ th quantile, the model predictions are expected to underpredict the realized return $\alpha=(1-q)$ percent of the time. A high number of exceptions implies that the model excessively underestimates the realized return. $\mathrm{f}$ the exception ratio at the $q$ th quantile is greater than $\alpha$ percent, this implies excessive underprediction of the realized return. If the number of exceptions is less than $\alpha$ percent at the $q$ th quantile, there is excessive overprediction of the realized return by the underlying model. Notice that the estimated return determines how much capital should be allocated for a given portfolio assuming that the investor has a short position in the market. Therefore, a high number of exceptions implies that the model signals less capital allocation and the portfolio risk is not properly hedged. In other words, the model increases the risk exposure by underpredicting it. On the other hand, exceptions excessively lower than the realized returns implies that the model signals a capital allocation more than necessary. In this case, the portfolio holder allocates more to liquidity and registers an interest rate loss. A regulatory body may prefer a model overpredicting the risk since the institutions will allocate more capital for regulatory purposes. Institutions would prefer a model underpredicting the risk, since they have to al- locate less capital for regulatory purposes, if they are using the
} 
Coverage tests suggested respectively by Kupiec (1995) and Christoffersen (1998) . These tests asses the adequacy of the models by considering the number of VaR exceptions, i.e. days when returns exceed VaR estimates. If the number of exceptions is less than the selected significance level would indicate, the system overestimates risk; on the contrary too many exceptions signal underestimation of risk. In particular the first test examines whether the frequency of exceptions over some specified time interval is in line with the selected significance level. A good VaR model produces not only the "correct" amount of exceptions but also exceptions that are independent each other, i.e. not clustered over time. Tests of conditional coverage take into account for the number of exceptions and when the exceptions occur. The tick loss function considered in order to run the tests is defined as Binary loss function (BLF). The aim of the BLF is to count the number of exceptions, that are verified when the loss is larger than the forecasted $V a R$ :

$$
B L F_{t+1}=\left\{\begin{array}{lll}
1 & \text { if } & R_{t+1}<\widehat{V a R}_{t+1 \mid t} \\
0 & \text { if } & R_{t+1} \geq \widehat{V a R}_{t+1 \mid t}
\end{array}\right.
$$

where $\widehat{V a R}_{t+1 \mid t}$ is the estimated VaR at time $t$ that refers to the period $t+1$.

The Likelihood Ratio test of unconditional coverage tests the null hypothesis that the true probability of occurrence of an exception over a given period is equal to $\alpha$ :

$$
\begin{array}{ll}
H_{0}: & p=\alpha \\
H_{1}: & p \neq \alpha
\end{array}
$$

where $\widehat{p}=\frac{n_{0}}{n_{1}+n_{0}}$ is the unconditional coverage (the empirical coverage rate) or the failure rate and $n_{0}$ and $n_{1}$ denote, respectively, the number of exceptions observed in the sample size and the number of non-exceptions.

The unconditional test statistic is given by:

$$
L R_{U C}=-2 \log \left(\frac{(1-\alpha)^{n_{1}} \alpha^{n_{0}}}{(1-\widehat{p})^{n_{1}} \widehat{p}^{n_{0}}}\right) \sim \chi^{2}(1)
$$

So, under the null hypothesis the significance level used to forecast VaRs and the empirical coverage rate are equal. The test of conditional coverage proposed by Christoffersen (1998)is an extended version of the previous one taking into consideration whether the probability of an exception on any day depends on the exception occurrence in the previous day. The loss function in constructed as in (27) and the log-likelihood testing framework is as in (28) including a separate statistic for independence of exceptions. Define the number of days

model only to meet the regulatory requirements. For this reason, the implemented capital allocation ratio is increased by the regulatory bodies for those models that consistently underpredict the risk 
when outcome $j$ occurs given that outcome $i$ occurred on the previous day as $n_{i j}$ and the probability of observing an exception conditional on outcome $i$ of the previous day as $\pi_{i}$. Summarizing:

$$
\pi_{0}=\frac{n_{01}}{n_{00}+n_{01}} \quad \pi_{1}=\frac{n_{11}}{n_{10}+n_{11}} \quad \pi=\frac{n_{01}+n_{11}}{n_{00}+n_{01}+n_{10}+n_{11}}
$$

The independence test statistic is given by:

$$
L R_{I N D}=-2 \log \left(\frac{(1-\pi)^{n_{00}+n_{10}} \pi^{n_{01}+n_{11}}}{\left(1-\pi_{0}\right)^{n_{00}} \pi_{0}^{n_{01}}\left(1-\pi_{1}\right)^{n_{10}} \pi_{1}^{n_{11}}}\right)
$$

Under the null hypothesis the first two probabilities in (29) are equal, i.e. the exceptions do not occur in cluster. Summing the statistics (28) and (30) the conditional coverage statistic is obtained, i.e. $L R_{C C}=L R_{U C}+L R_{I N D}$ and it is distributed as a $\chi^{2}$ with two degrees of freedom since two is the number of possible outcomes in the sequence in (27). In order to avoid the possibility that the models considered pass the joint test but fail either the coverage or the independence test I choose to run $L R_{C C}$ and also its decomposition in $L R_{U C}$ and $L R_{I N D}$.

\section{Data and Empirical results}

\subsection{Data}

In order to assess which volatility measure and, in turn, which sampling frequency is better in terms of VaR forecasting and accuracy, I use S\&P 500 index from 5 Jan.1996 to 30 Dec.2005 both for daily and high frequency samples.

The total number of trading days is equal to 2516 which coincides with the number of daily returns. In the top panel of Figure 1 the level of the S\&P 500 index is presented. The corresponding daily returns are displayed in the bottom panel of Figure 1.

Given the literature on the effects on microstructure noise of estimates of RV and the forecast performance of RV models based on different sampling frequency, I use 5-minutes data for a total of 197,689 observations and I compute 5-minutes intraday returns as the log-difference of the closing prices in two subsequent periods of time. The daily returns are computed taking the last closing prices in each trading day. The range volatility at each date is calculated as scaled log-difference between the highest and the lowest price in a trading day over all the prices recorded. 

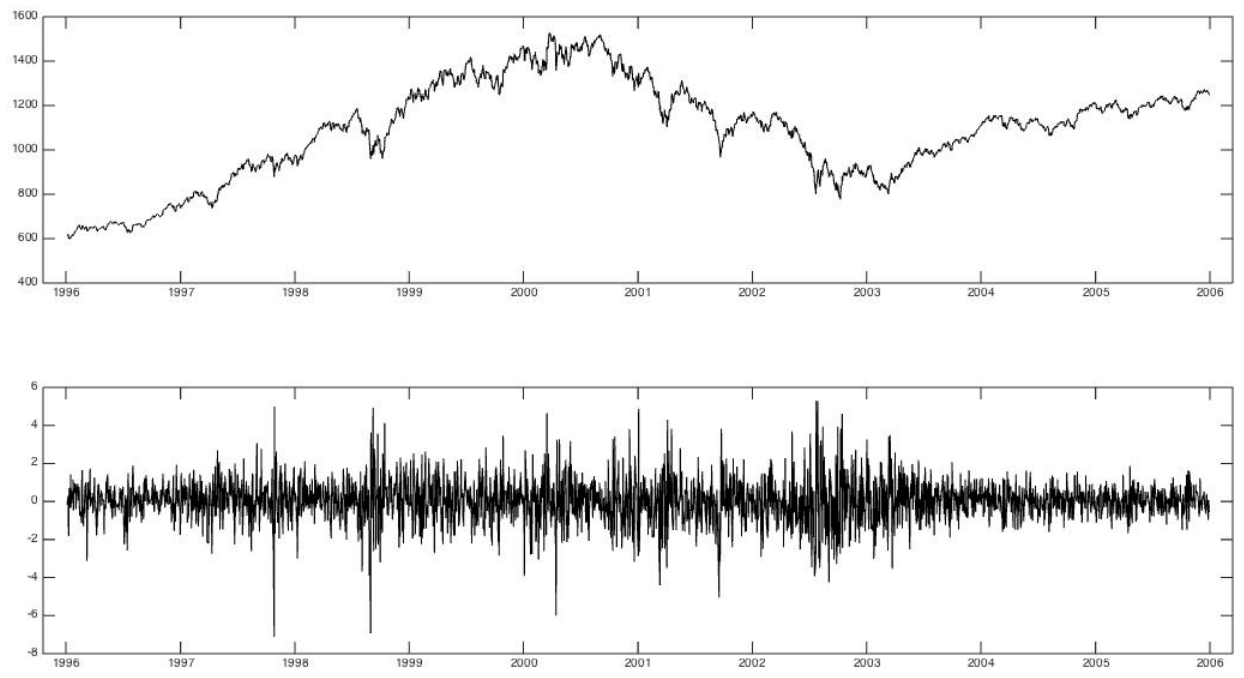

Figure 1: Top: daily S\&P 500 index from 5 Jan.1996 to 30 Dec.2005. The horizontal axis corresponds to time while the vertical axis displays the value of the index. Bottom: daily S\&P 500 percentage returns calculated by $r_{t}=\log \left(p_{t} / p_{t-1}\right)$, where $p_{t}$ is the value of the index at time $t$.

Table 1: Summary Statistics

\begin{tabular}{rrrrrrr}
\hline & $R_{t}$ & $\left.R_{t} / \sqrt{(} R V_{t}\right)$ & $R V_{t}$ & $B V_{t}$ & $J V_{t}$ & $R A_{t}$ \\
\hline \hline Mean & 0.0279 & 0.1378 & 0.8250 & $7.93 \mathrm{E}-05$ & $3.15 \mathrm{E}-06$ & $9.70 \mathrm{E}-05$ \\
St. Dev. & 1.1520 & 1.3138 & 1.0097 & $9.85 \mathrm{E}-05$ & $1.00 \mathrm{E}-05$ & $1.52 \mathrm{E}-04$ \\
Skewness & -0.0951 & 0.253 & 4.8721 & 4.8786 & 19.9283 & 7.1671 \\
Kurtosis & 5.9165 & 2.8505 & 39.1013 & 39.3401 & 659.3967 & 84.1687 \\
Min & -7.1127 & -3.6092 & 0.0281 & 0.0281 & 0 & 0.0206 \\
Max & 5.3080 & 4.7161 & 11.890 & 11.890 & 3.6200 & 25.931 \\
\hline \hline
\end{tabular}

Notes: the rows report the sample mean, standard deviation, skewnwss, kurtosis, sample minimum and maximum for the daily returns $\left(R_{t}\right)$, the standardized daily returns $\left(R_{t} / \sqrt{\left(R V_{t}\right)}\right)$ the daily realized volatility $\left(R V_{t}\right)$, the daily bipower variation $\left(B V_{t}\right)$, the daily jump component $\left(J V_{t}\right)$ and the daily range estimator $\left(R A_{t}\right)$. Retuns are expressed in percentage.

Table 1 reports the descriptive statistics of S\&P 500 index for $R V_{t}$ and its decomposition 
in $B V_{t}$ and $J V_{t}$. In particular $J V_{t}$ is computed as $\max \left\{R V_{t}-B V_{t}, 0\right\} .{ }^{21}$ and Range measure. A number of interesting features are founded. Firstly, returns exhibit negative asymmetry and leptokurtosis. As shown in Andersen et al. (2007) the daily returns standardized with respect to the square root of the ex-post realized volatility are closed to Gaussian. In fact its mean and asymmetry are close to zero, its variance is close to one while its kurtosis is near to 3. This result is clear from Figure 2 in which the empirical density distribution is plotted with the normal density distribution for $R_{t} / \sqrt{R V_{t}}$. Moreover if I compare $R V_{t}$ and $B V_{t}$ the latter is less noisy than the former, considering the role of jumps. Finally, jump process shows any Gaussian feature ${ }^{22}$.

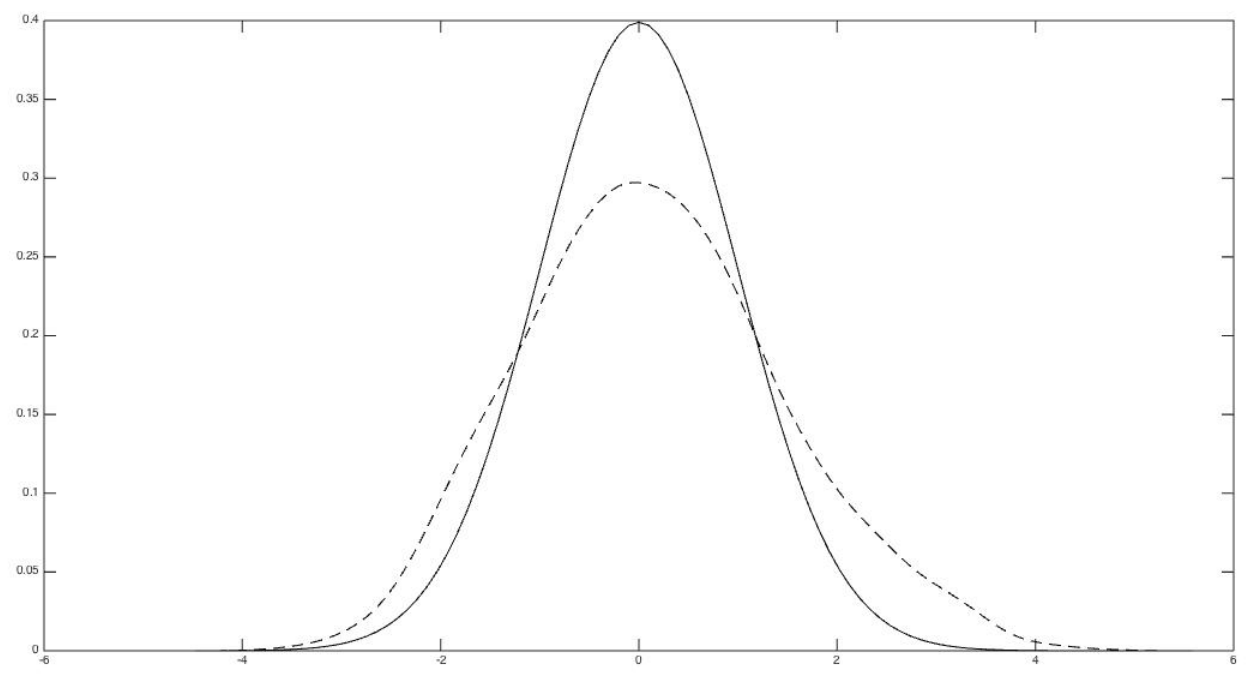

Figure 2: The graph displays the density distributions, i.e. empirical (dashed lines) vs normal (solid lines), for the daily returns standardized with respect to the square root of the ex-post realized volatility computed for the and S\&P500 stock index based on 5-minute returns.

Figure 3 shows the plot of $R V_{t}, B V_{t}, J V_{t}$ and $R A_{t}$ estimators. It is evident $R V_{t}, B V_{t}$ and $J V_{t}$ follow a similar pattern and $J V_{t}$ tends to be higher when $R V_{t}$ is higher even if its maximum does not correspond to $R V_{t}$ (and $B V_{t}$ ) maximum. The jumps exhibit a relatively small degree of persistence as consequence of the clustering effect. Moreover $R A_{t}$ estimator follows the same pattern of $R V_{t}$ assuming that both of them are ex-post volatility measures.

\footnotetext{
${ }^{21}$ The summary statistics of the continuous and disontinuous components computed according to Andersen et al. (2007) and Corsi et al. (2010) are not reported because are very similar to those presented in Table 1.

${ }^{22}$ In particular, jumps computed according to (6)exhibit a higher mean with respect to those computed according to (4), given that the former exploits the possibility of consecutive jumps.
} 

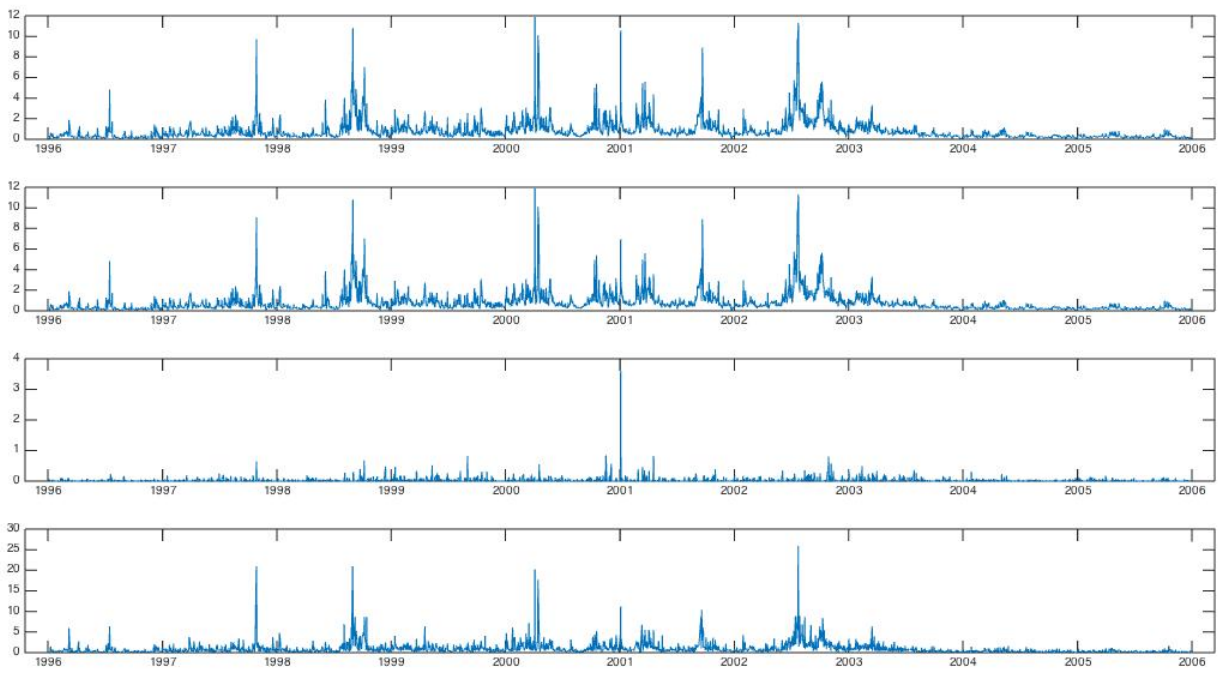

Figure 3: Top: $R V_{t}$ computed using 5- minutes data from 5 Jan.1996 to 30 Dec.2005. The horizontal axis corresponds to time while the vertical axis displays the value RV. Second: $B V_{t}$ computed using 5- minutes data from 5 Jan.1996 to 30 Dec.2005. Third: $J V_{t}=\max \left\{R V_{t}-\right.$ $\left.B V_{t}, 0\right\}$ is computed using 5- minutes data from 5 Jan.1996 to 30 Dec.2005. Bottom: Range estimator computed using daily data from 5 Jan.1996 to 30 Dec.2005.

\subsubsection{Estimation results based on daily data}

Table A1, reported in Appendix A, provides parameter estimates for both GARJI and ARJI model applied to S\&P500. The parameter estimates are presented separating the diffusion component from the jump component. First, both parameters $\rho$ and $\gamma$ are significantly different from zero. The former represents the persistence of the arrival process of jumps that is quite high for both models which implies the presence of jump clustering. The latter, $\gamma$, measures the change in the conditional forecast of the number of jumps due to the last days information. The significance of these two parameters suggests that the arrival process of jumps can deviate from its unconditional mean. The implied unconditional jump intensity is 0.8727 while the average variance due to jumps is equal to 0.5516 which means that the index is volatile. This result is confirmed by the average proportion of conditional variance explained by jumps that is equal to 0.3068 , jumps explained almost the $23 \%$ of the total returns variance. Moreover the jump size mean $\theta$ is negative for both model and the most interesting feature is that it affects conditional skewness and conditional kurtosis. The sign of $\theta$ indicates that large negative return realizations due to jumps are associated with an immediate increase in the variance explaining the contemporaneous leverage effect: when jumps are realized they tend to have a negative effect on returns. In particular the average conditional skewness is equal to -0.2766 while the average conditional kurtosis is equal to 
3.2814. Furthermore the feedback coefficient $g\left(\Lambda, \mathcal{F}_{t-1}\right)$ tends to be smaller when at least one jump occurs because the total innovation is larger after jumps . Consider the first column of Table A1, the feedback coefficient associated with good news and no jump is equal to 0.0005 and it increases if one jump occurs, i.e. 0.0010. If no jumps occur and in presence of bad news the coefficient is equal to 0.0411 and it is equal to 0.0348 in case of bad news if one jump occurs. These results provide evidence for the asymmetric effect of good and bad news and they show that the asymmetry associated to bad news is more important in the absence of jumps, namely for normal innovations. In fact the difference between the coefficient estimates for both good and bad news in the case of no jumps and one jump are quite similar. This means that news associated with jump innovations is incorporated more quickly into current prices. The second column of Table A1 presents the estimated parameters for the model with $\alpha_{j}=\alpha_{a}=\alpha_{a, j}=0$. With this specification and through the LR test it is possible to understand if the asymmetric effect of good versus bad news is statistically significant: the asymmetric news effect is statistically significant.

\subsubsection{Estimation results based on high frequency data}

All the estimates presented in Table A2 and Table A3 in Appendix A, are computed employing the OLS method over the entire sample period, i.e. from 5 Jan. 1996 to 30 Dec. 2005, for the S\&P500 index. Table A2 shows the results for the models presented in Section 3.2 for models based on RV, its decomposition in BV and JV and the cascade structure for the leverage effect. The coefficients of the continuos component expressed as daily, weekly and monthly measures, respectively $\beta_{1}, \beta_{2}$ and $\beta_{3}$ are significants in all models. Moreover jump components appear to be fundamental to forecast one step ahead volatility; the predictive power is higher for those specifications that allows for RV decomposed in its continuous and discontinuous components, regardless the identified method used for jump magnitude. Furthermore, the estimates for the aggregate variables representing the asymmetric responses of volatility to negative returns are negatives (as expected) and significants and the predictive power increases adding leverage regressors.

This finding confirms the different reaction of daily volatility to negative returns. The estimates of the forecasting models based on the Range estimator are reported in Table A3. The coefficients of the HAR specification are statistically significant; these results imply a heterogenous structure also for RA volatility measure. the highest predictive power is recorded

for the L-HAR model. Indeed also in this case, the heterogenous structure in the leverage effect has an important role in predicting future volatility. 


\subsection{VaR accuracy results}

To asses the models capability of predicting future volatility, I report the results of the Kupiec (1995) and the Christoffersen (1998) tests described in the Section 4. Both tests address the accuracy of VaR models and their results interpretation give insigths into volatility models useful to risk managers and supervisory authorities. The tests are computed for both models based on HF data and on daily data. In evaluating models performance, the available sample is divided into two subsamples: in-sample period is equal to 1677 observation, around $2 / 3$ of the total sample, while the out-of-sample period is around $1 / 3$ of the total sample, equal to 839 observations. A moving window procedure is used to implement and evaluate the models according to the tests. After estimating the alternative VaR models, the one-day-ahead VaR estimate is computing using the in-sample period. Then the in-sample period is moved forward by one period and the estimation is runned again. This procedure is repeated step by step for the remaining 839 days, until the end of the sample. For both tests the expected number of exceedances is chosen equal to $10 \%, 5 \%$ and $1 \%$ level. The results, displayed in Table 2, show that for all models presented in the Section 3.2 the unconditional coverage test $\left(L_{U C}\right)$ rejects at $10 \%, 5 \%$ and $1 \%$ significance levels the null of an accurate interval forecast. This means that the actual number of violations is statistically different from the expected fraction, i.e. $10 \%, 5 \%$ and $1 \%$ levels. The only exception is represented by the AR(8) model at $1 \%$ significance level. This VaR model predicts the actual fraction of violation that is equal to the expected fraction (1\%). Moreover the Christoffersen (1998) test reject the null for all models and for all confidence level assumed, excepted for the AR(8) model at $1 \%$. Since the null hypothesis of the $L R_{C C}$ test is the independence of the observed violations, this finding can be associated with the presence of violations clustering ascibable to volatility persistence. The same conclusions can be done also for models based on RA measure as showed in Table 3, even for the AR(8) model (at 1\%). This is in line with the interpretation of RA. So, exploring informations of the intraday activity using daily data gives a similar VaR accurancy with respect to informations obtained sampling data at higher frequency. These results highlight an interesting aspect related to HF based models capability of predicting tail events. All models reject the null in both tests and some models present the same value for the test statistcs. For example, from Table 2 LHARC-Jumps, LHAR-CV-JV and LHAR-C-J present the same conclusions for $L R_{U C}$ and $L R_{C C}$. These models are characterized by the same heterogeneous structure for continuous and discontinuous volatiltiy components and leverage effect ${ }^{23}$.

\footnotetext{
${ }^{23}$ The leverage variables are the same in all models based on high frequency data. They are constructed aggregating the past negative returns in the same time span obtaining a cascade structure.
} 


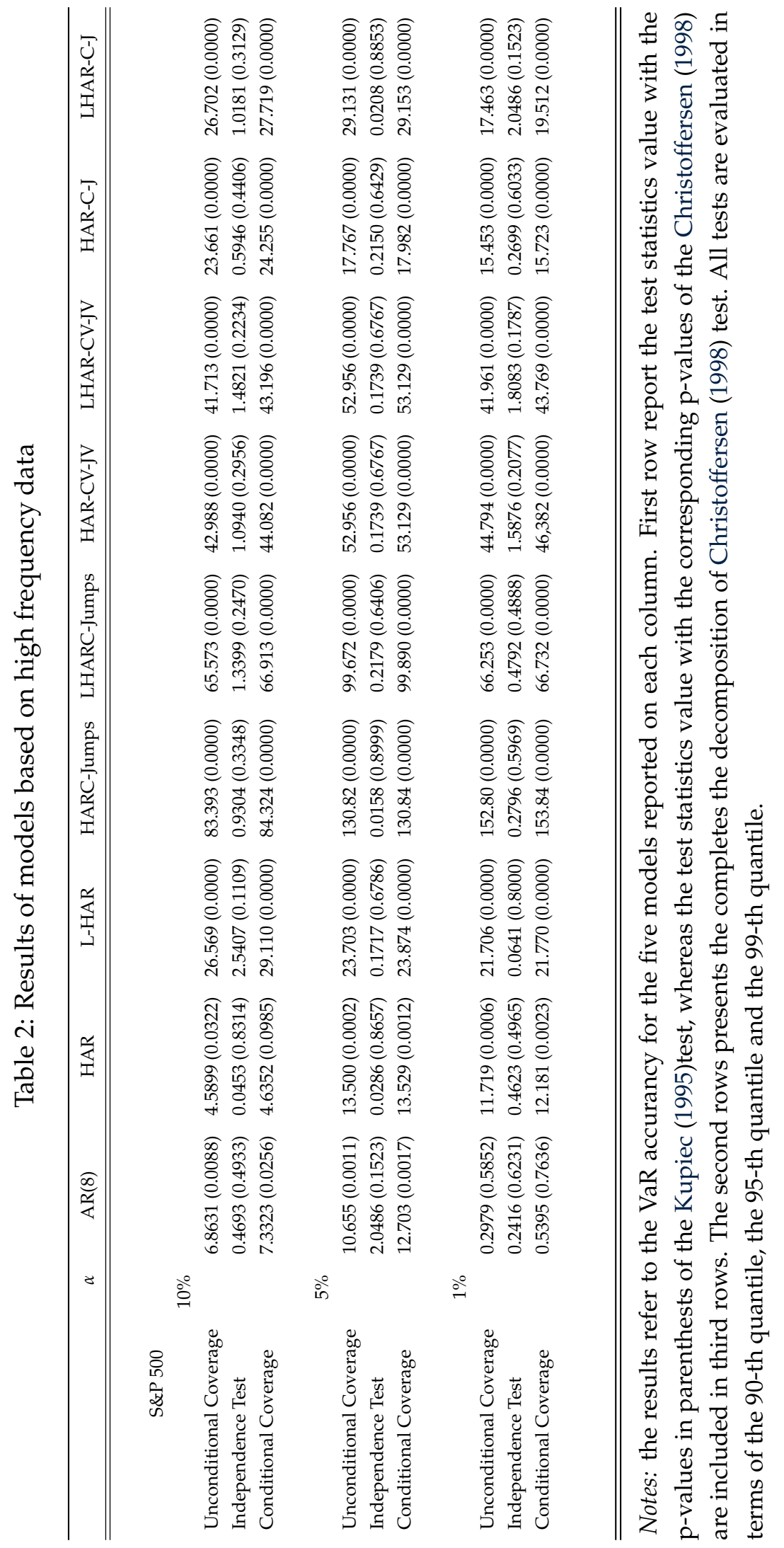


These models differ in the strategy adopted to detect spikes in the price trajectories. LHARJumps model accounts for jumps as difference between RV and BV, LHAR-CV-JV detects significant jumps according to the procedure presented by Andersen et al. (2007) while LHARC-J asses consecutive jumps according to the test proposed by Corsi et al. (2010).

Table 3: Results of models based on Range estimator

\begin{tabular}{lcccc}
\hline & $\alpha$ & AR(8) & HAR & L-HAR \\
\hline \hline S\&P 500 & & & & \\
& $10 \%$ & & & \\
Unconditional Coverage & & $21.008(0.0000)$ & $29.905(0.0000)$ & $46.909(0.0000)$ \\
Independence Test & & $3.6806(0.0551)$ & $0.2033(0.6520)$ & $0.7011(0.4024)$ \\
Conditional Coverage & & $24.689(0.0000)$ & $30.108(0.0000)$ & $47.610(0.0000)$ \\
& & & & \\
& $5 \%$ & & & \\
Unconditional Coverage & & $13.332(0.0002)$ & $36.429(0.0000)$ & $60.244(0.0000)$ \\
Independence Test & & $0.3588(0.5492)$ & $0.3985(0.5279)$ & $0.0540(0.8162)$ \\
Conditional Coverage & & $13.691(0.0011)$ & $36.827(0.0000)$ & $60.298(0.0000)$ \\
& & & & \\
& $1 \%$ & & & \\
Unconditional Coverage & & $0.7578(0.3840)$ & $66.253(0.0000)$ & $41.962(0.0000)$ \\
Independence Test & & $0.0865(0.7686)$ & $0.4793(0.4888)$ & $1.8083(0.1787)$ \\
Conditional Coverage & $0.8443(0.6556)$ & $66.732(0.0000)$ & $43.769(0.0000)$
\end{tabular}

Notes: the results refer to the VaR accurancy for the three models based on Range estimator reported on each column. First row report the test statistics value with the p-values in parenthests of the $\mathrm{Ku}$ piec (1995)test, whereas the test statistics value with the corresponding p-values of the Christoffersen (1998) are included in third rows. The second rows presents the completes the decomposition of Christoffersen (1998) test. All tests are evaluated in terms of the 90-th quantile, the 95-th quantile and the 99 -th quantile.

Even if all three models offer different one step ahead VaR forecasts, none of them can be interpreted as an improvement in terms of prediction accurancy of extreme events. In same cases the fraction of violations detected is the same, producing the same test statistics. Another interesting comparison can be done between AR(8) and HAR models. The heterogeneous structure for volatility is introducted by Corsi and Renó (2009) to approximate serial dependence in volatility using a model that is easier to implement than a pure long memory model. This model reproduces the memory persistence exploiting the asymmetric propaga- 
tion of volatility between long and short time horizons generated by the actions of different types of market participants according to the Heterogeneous Market Hypothesis. According to the results shown in Table 2 HAR volatility model does not provide a better accuracy of predicting tail events than the AR(8) model. The cascade structure of the HAR model is not sufficient to reproduce more accurate VaR estimates. The AR(8) model is even more accurate than the HAR since it predicts extreme events and independent violations, at least at $1 \%$ level.

Table 4: Results for models based on data sampled at daily frequency

\begin{tabular}{lccccc}
\hline & $\alpha$ & GARJI & ARJI & GARCH- $t$ & Beta-t-GARCH \\
\hline \hline S\&P 500 & & & & & \\
& $10 \%$ & & & & \\
Unconditional Coverage & & $9.0010(0.0027)$ & $10.608(0.0011)$ & $24.971(0.0000)$ & $24.971(0.0000)$ \\
Independence Test & & $1.9256(0.1652)$ & $1.1724(0.2789)$ & $4.7647(0.0290)$ & $4.7647(0.0290)$ \\
Conditional Coverage & & $10.927(0.0042)$ & $11.780(0.0028)$ & $29.736(0.0000)$ & $29.736(0.0000)$ \\
& & & & & \\
Unconditional Coverage & & $9.4524(0.0021)$ & $14.815(0.0001)$ & $6.3469(0.0118)$ & $6.3469(0.0118)$ \\
Independence Test & & $4.6974(0.0302)$ & $2.9323(0.0868)$ & $1.1706(0.2793)$ & $1.1706(0.2793)$ \\
Conditional Coverage & & $14.149(0.0008)$ & $17.747(0.0001)$ & $7.5175(0.0233)$ & $7.5175(0.0233)$ \\
& & & & & \\
& $1 \%$ & & & & \\
Unconditional Coverage & $4.6314(0.0314)$ & $7.0781(0.0078)$ & $0.2432(0.6219)$ & $0.0177(0.8942)$ \\
Independence Test & $0.0216(0.8833)$ & $0.0096(0.9221)$ & $0.1179(0.7313)$ & $0.1542(0.6945)$ \\
Conditional Coverage & $4.6530(0.0976)$ & $7.0877(0.0289)$ & $0.3611(0.8348)$ & $0.1719(0.9176)$
\end{tabular}

Notes: The results refer to the VaR accurancy for the GARJI, ARJI, GARCH- $t$ and Beta- $t$ models. All of them are daily based models with different assumption about the distribution of conditional returns. First row report the test statistics value with the p-values in parenthests of the Kupiec (1995)test, whereas the test statistics value with the corresponding p-values of the Christoffersen (1998) are included in third rows. The second rows presents the completes the decomposition of Christoffersen (1998) test. All tests are evaluated in terms of the 90-th quantile, the 95-th quantile and the 99-th quantile.

Different conclusions can be derived from Table 4. GARJI model does not reject the null for both $L R_{U C}$ and $L R_{C C}$ at $1 \%$ significance level. This finding can be interpreted in favour to forecasting volatility models, and in turn VaR models, based on daily data. Daily data are preferred to the use of HF data since the former offer more accurate VaR measures. Table 4 also records the $10 \%, 5 \%$ and $1 \% \mathrm{VaRs}^{\prime}$ results for GARCH-t and Beta-t-GARCH models. 
Both of them are constructed on the idea that the standardized returns present fatter tails than a normal distribution. For this reason VaR estimates are computed assuming a $t$-Student distribution for standardized returns. Both models lead to the same outcome: they pass VaR tests only at $1 \%$ level. The nice thing is that the magnitude of all test statistics are equal at $10 \%$ and $5 \%$ level. A possible motivation could be recognized in the fact that in the limit, as the degrees of freedom go to infinity, the Beta-t-GARCH model coincide with the standard GARCH- $t$ model. Given this last comparison there is no difference in terms of VaR accurancy between a model that allows for an explicit jump component and a fat-tail distributional assumption. Neverthless, providing jumps gives to a risk manager important informations about the market response to outside news.

\section{Conclusions}

This paper assesses the economic value of different forecasting volatility models, in particular by comparing the performances of HF-data and daily-data models in a VaR framework. In so doing, two key assumptions are introduced: jumps in price and leverage effect in volatility dynamics. I consider various specifications of HF-data models for volatility forecast, which differs along three main dimensions: different time-horizons for investors, separation of continuous and discontinuous volatility components and, finally, a cascade dynamic for the leverage effect. I also consider different variants of the daily-data models, in form of GARJI models either with or without an asymmetric effect of news on volatility, as well as in form of two fat-tails models, namely the GARCH- $t$ and the Beta- $t$ GARCH models. All these models are compared with a correspondent and equivalent model, based on the Range volatility measure; the latter is expected to estimate a level of volatility which is intermediate with respect to those measured by HF-data and daily-data models. This analysis highlights two important issues. First, it stresses the importance of the sampling frequency for data needed in economic applications such as the VaR measurement. Second, it emphasizes the strict relationship between VaR measures and the type of model used to forecast volatility. In sum, daily-data models are preferred to HF-data models: all volatility forecasting models based on HF-data do not pass the VaR tests (at 10\%, 5\% and 1\%, except for the AR (8) model at 1\% level). On the contrary, the GARJI model does not reject the null for both the Unconditional and Conditional Coverage tests (at 1\% significance level). Moreover, it seems that the accurancy of the VaR measure does not significantly improve when introducing both an explicit jump component and a fat-tail distribution in forecasting volatility models, independently on the type of data (HF or daily) required by the model. However, introducing jumps allows risk managers to have relevant information on the market reaction to outside news. Hence, it seems plausible to argue that a more sophisticated model for volatility might, in principle, improve the accuracy of the VaR results. In partic- 
ular for the case of HF-data models, conditional normality does not appear as a reasonable assumption: based on the VaR results obtained in this paper, these models do not manage to make the tails of the conditionally Gaussian distribution fat enough to match the unconditional distribution, even when jumps contribution is accounted for. Such conjectures might be tested by using a model in which the future values of RV is explained based on a larger set of explanatory variables, including, for instance, the level of market activity. An alternative option may be represented by a model which assumes a distribution of asset returns that is fatten than the Normal, even if RV are model-free estimators and standardized returns are Normal distributed. All these questions are left for future research, together with a possible replication of the same analysis proposed by this paper, but performed on data for a different time period or different asset returns. Indeed, the normal distribution of standardized returns should not be confirmed when using different samples: in this case, VaR forecasting might require an empirical distribution for standardized returns, fatten than the Normal. 


\section{Appendix A}

Table A1: GARJI and ARJI models estimates

\begin{tabular}{lccc}
\hline Process & Parameters & S\&P 500 \\
\hline \hline \multirow{2}{*}{ Diffusion } & & GARJI & ARJI \\
& $\mu$ & $0.0106(1.9839)$ & $0.0153(2.1142)$ \\
$\omega$ & $0.0036(0.0005)$ & $0.0034(0.0005)$ \\
$\alpha$ & $-7.7048(0.4332)$ & $-4.7623(0.3063)$ \\
$\alpha_{j}$ & $0.8096(0.7538)$ & - \\
$\alpha_{a}$ & $4.5131(0.4213)$ & - \\
& $\alpha_{a, j}$ & $-0.9776(0.7204)$ & - \\
$\beta$ & $0.9696(0.0002)$ & $0.9787(0.0000)$ \\
Jump & & & \\
& $\lambda_{0}$ & $0.0211(0.0039)$ & $0.0229(0.0052)$ \\
$\rho$ & $0.9758(0.0025)$ & $0.9757(0.0030)$ \\
$\gamma$ & $0.5262(0.0501)$ & $0.4792(0.0641)$ \\
& $\theta$ & $-0.9895(0.3985)$ & $-0.9793(0.4501)$ \\
$\delta^{2}$ & $0.0005(0.0000)$ & $0.0000(0.0000)$ \\
\hline Log-likelihood & & -3570.8 & -3574.4 \\
\hline \hline
\end{tabular}

Notes: ARJI model is obtained assuming $\alpha_{j}=\alpha_{a}=\alpha_{a, j}=0$. Standard errors are in parenthesis. 


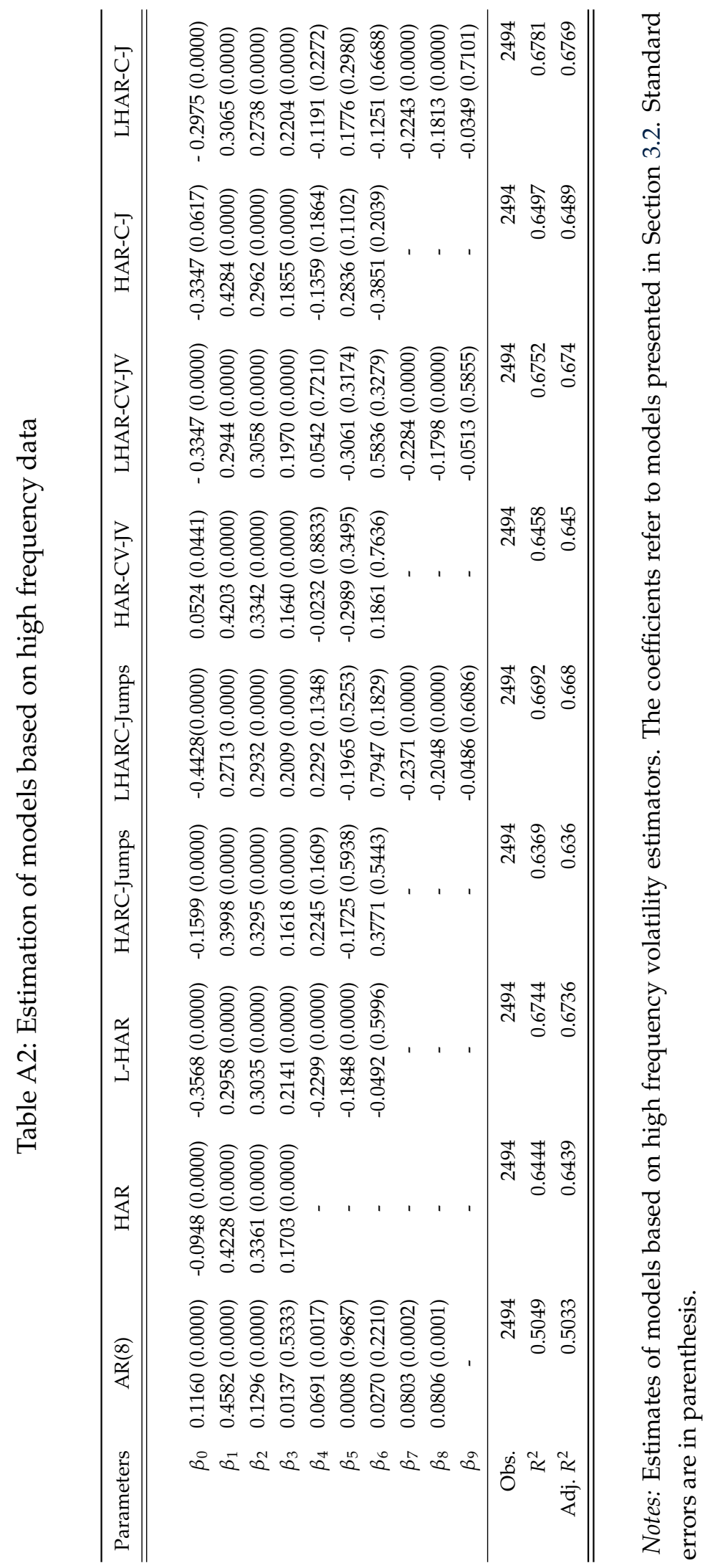


Table A3: Estimation of models based on Range estimator

\begin{tabular}{rrrr}
\hline Parameters & AR(8) & HAR & L-HAR \\
\hline \hline$\beta_{0}$ & $0.2347(0.0000)$ & $-02601(0.0000)$ & $-0.8119(0.0000)$ \\
$\beta_{1}$ & $0.2542(0.0000)$ & $0.1147(0.0000)$ & $-0.0279(0.2066)$ \\
$\beta_{2}$ & $0.1141(0.0000)$ & $0.4502(0.0000)$ & $0.3013(0.0000)$ \\
$\beta_{3}$ & $0.0986(0.5333)$ & $0.3209(0.0000)$ & $0.3530(0.0000)$ \\
$\beta_{4}$ & $0.0141(0.4953)$ & - & $-0.3011(0.0000)$ \\
$\beta_{5}$ & $0.0620(0.0027)$ & - & $-0.3765(0.0000)$ \\
$\beta_{6}$ & $0.0078(0.7034)$ & & $-0.3509(0.0160)$ \\
$\beta_{7}$ & $0.0825(0.0002)$ & - & - \\
$\beta_{8}$ & $0.1244(0.0000)$ & - & 2494 \\
Obs. & 2494 & & 2494 \\
$R^{2}$ & 0.2592 & 0.3914 & 0.4337 \\
Adj. $R^{2}$ & 0.2568 & 0.3907 & 0.4323 \\
\hline \hline
\end{tabular}

Notes: Estimates of models based on Range volatility estimator. The coefficients refer to models presented in Section 3.2. Standard errors are in parenthesis.

\section{References}

Aït-Sahalia, Y. and J. Jacod (2010). Analyzing the spectrum of asset returns: Jump and volatility components in high frequency data. Technical report, National Bureau of Economic Research.

Aït-Sahalia, Y. and L. Mancini (2008). Out of sample forecasts of quadratic variation. Journal of Econometrics 147(1), 17-33.

Aït-Sahalia, Y., P. A. Mykland, and L. Zhang (2005). How often to sample a continuous-time process in the presence of market microstructure noise. Review of Financial studies 18(2), 351-416.

Aït-Sahalia, Y., P. A. Mykland, and L. Zhang (2011). Ultra high frequency volatility estimation with dependent microstructure noise. Journal of Econometrics 160(1), 160-175.

Alizadeh, S., M. W. Brandt, and F. X. Diebold (2002). Range-based estimation of stochastic volatility models. The Journal of Finance 57(3), 1047-1091.

Andersen, T. G. and T. Bollerslev (1998). Answering the skeptics: Yes, standard volatility models do provide accurate forecasts. International economic review, 885-905. 
Andersen, T. G., T. Bollerslev, P. F. Christoffersen, and F. X. Diebold (2012). Financial risk measurement for financial risk management. Technical report, National Bureau of Economic Research.

Andersen, T. G., T. Bollerslev, and F. X. Diebold (2007). Roughing it up: Including jump components in the measurement, modeling, and forecasting of return volatility. The Review of Economics and Statistics 89(4), 701-720.

Andersen, T. G., T. Bollerslev, F. X. Diebold, and H. Ebens (2001a). The distribution of realized stock return volatility. Journal of financial economics 61(1), 43-76.

Andersen, T. G., T. Bollerslev, F. X. Diebold, and P. Labys (2003). Modeling and forecasting realized volatility. Econometrica 71(2), 579-625.

Andersen, T. G., T. Bollerslev, F. X. Diebold, and C. Vega (2002). Micro effects of macro announcements: Real-time price discovery in foreign exchange. Technical report, National bureau of economic research.

Andersen, T. G., T. Bollerslev, P. Frederiksen, and M. Ørregaard Nielsen (2010). Continuoustime models, realized volatilities, and testable distributional implications for daily stock returns. Journal of Applied Econometrics 25(2), 233-261.

Barndorff-Nielsen, O. E. (2002). Econometric analysis of realized volatility and its use in estimating stochastic volatility models. Journal of the Royal Statistical Society: Series B (Statistical Methodology) 64(2), 253-280.

Barndorff-Nielsen, O. E. and N. Shephard (2004). Power and bipower variation with stochastic volatility and jumps. Journal of financial econometrics 2(1), 1-37.

Bates, D. S. (1996). Jumps and stochastic volatility: Exchange rate processes implicit in deutsche mark options. Review of financial studies 9(1), 69-107.

Bates, D. S. (2000). Post-' 87 crash fears in the s\&p 500 futures option market. Journal of Econometrics 94(1), 181-238.

Bollerslev, T. (1986). Generalized autoregressive conditional heteroskedasticity. Journal of econometrics 31(3), 307-327.

Bollerslev, T. and V. Todorov (2011). Tails, fears, and risk premia. The Journal of Finance 66(6), 2165-2211.

Brownlees, C. T. and G. M. Gallo (2010). Comparison of volatility measures: a risk management perspective. Journal of Financial Econometrics 8(1), 29-56. 
Chan, W. H. and J. M. Maheu (2002). Conditional jump dynamics in stock market returns. Journal of Business E Economic Statistics 20(3), 377-389.

Chiu, C.-L., M.-C. Lee, and J.-C. Hung (2005). Estimation of value-at-risk under jump dynamics and asymmetric information. Applied Financial Economics 15(15), 1095-1106.

Christensen, K., R. C. Oomen, and M. Podolskij (2014). Fact or friction: Jumps at ultra high frequency. Journal of Financial Economics 114(3), 576-599.

Christoffersen, P., R. Elkamhi, B. Feunou, and K. Jacobs (2009). Option valuation with conditional heteroskedasticity and nonnormality. Review of Financial studies, hhp078.

Christoffersen, P. F. (1998). Evaluating interval forecasts. International economic review, 841862.

Christoffersen, P. F. and F. X. Diebold (2000). How relevant is volatility forecasting for financial risk management? Review of Economics and Statistics 82(1), 12-22.

Clements, M. P., A. B. Galvão, and J. H. Kim (2008). Quantile forecasts of daily exchange rate returns from forecasts of realized volatility. Journal of Empirical Finance 15(4), 729-750.

Corsi, F. (2009). A simple approximate long-memory model of realized volatility. Journal of Financial Econometrics, nbp001.

Corsi, F., D. Pirino, and R. Reno (2010). Threshold bipower variation and the impact of jumps on volatility forecasting. Journal of Econometrics 159(2), 276-288.

Corsi, F. and R. Renó (2009). Har volatility modelling with heterogeneous leverage and jumps. Available at SSRN 1316953.

Creal, D., S. J. Koopman, and A. Lucas (2013). Generalized autoregressive score models with applications. Journal of Applied Econometrics 28(5), 777-795.

Diebold, F. X. and R. S. Mariano (2012). Comparing predictive accuracy. Journal of Business E economic statistics.

Duan, J.-C. et al. (1995). The garch option pricing model. Mathematical finance 5(1), 13-32.

Duan, J.-C., P. Ritchken, and Z. Sun (2006). Approximating garch-jump models, jumpdiffusion processes, and option pricing. Mathematical Finance 16(1), 21-52.

Engle, R. F. (1982). Autoregressive conditional heteroscedasticity with estimates of the variance of united kingdom inflation. Econometrica: Journal of the Econometric Society, 987-1007.

Engle, R. F. and S. Manganelli (2004). Caviar: Conditional autoregressive value at risk by regression quantiles. Journal of Business E Economic Statistics 22(4), 367-381. 
Gençay, R., F. Selçuk, and A. Ulugülyağci (2003). High volatility, thick tails and extreme value theory in value-at-risk estimation. Insurance: Mathematics and Economics 33(2), 337-356.

Ghysels, E., P. Santa-Clara, and R. Valkanov (2004). The midas touch: Mixed data sampling regression models. Finance.

Giot, P. and S. Laurent (2004). Modelling daily value-at-risk using realized volatility and arch type models. journal of Empirical Finance 11(3), 379-398.

Hansen, P. R. and A. Lunde (2006). Realized variance and market microstructure noise. Journal of Business E Economic Statistics 24(2), 127-161.

Harvey, A. and A. Luati (2014). Filtering with heavy tails. Journal of the American Statistical Association 109(507), 1112-1122.

Heston, S. L. (1993). A closed-form solution for options with stochastic volatility with applications to bond and currency options. Review of financial studies 6(2), 327-343.

Heyde, C., S. Kou, and X. Peng (2006). What is a good risk measure: Bridging the gaps between data, coherent risk measures and insurance risk measures. Preprint, Columbia University.

Huang, X. and G. Tauchen (2005). The relative contribution of jumps to total price variance. Journal of financial econometrics 3(4), 456-499.

Hung, J.-C., M.-C. Lee, and H.-C. Liu (2008). Estimation of value-at-risk for energy commodities via fat-tailed garch models. Energy Economics 30(3), 1173-1191.

Kupiec, P. H. (1995). Techniques for verifying the accuracy of risk measurement models. THE J. OF DERIVATIVES 3(2).

Maheu, J. M. and T. H. McCurdy (2004). News arrival, jump dynamics, and volatility components for individual stock returns. The Journal of Finance 59(2), 755-793.

Merton, R. C. (1976). Option pricing when underlying stock returns are discontinuous. Journal of financial economics 3(1), 125-144.

Müller, U. A., M. M. Dacorogna, R. D. Davé, R. B. Olsen, O. V. Pictet, and J. E. von Weizsäcker (1997). Volatilities of different time resolutions-analyzing the dynamics of market components. Journal of Empirical Finance 4(2), 213-239.

Parkinson, M. (1980). The extreme value method for estimating the variance of the rate of return. Journal of Business, 61-65. 
Todorov, V. and G. Tauchen (2011). Volatility jumps. Journal of Business E Economic Statistics 29(3), 356-371.

Wu, L. (2003). Jumps and dynamic asset allocation. Review of Quantitative Finance and Accounting 20(3), 207-243. 


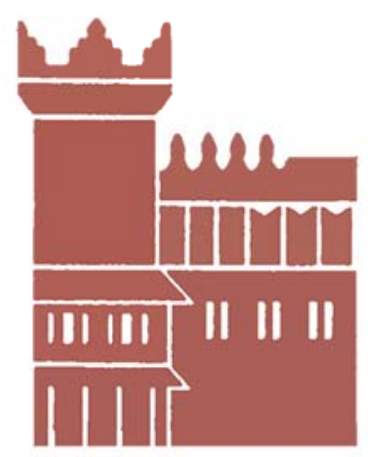

Alma Mater Studiorum - Università di Bologna DEPARTMENT OF ECONOMICS

Strada Maggiore 45

40125 Bologna - Italy

Tel. +39051 2092604

Fax +390512092664

http://www.dse.unibo.it 